\title{
Some Points in the Causation of Pelvic Suppuration.*
}

\author{
By G. Rothwell Adam, M.D. (Melb.), M.B., C.M. (Edin.), Lecturer on \\ Obstetrics and Gynacology, University of Melbourne; Hon. \\ Surgeon, Women's Hospital.
}

Is bringing before this section so important a subject as pelvic suppuration, I must crave permission, at the outset, to extend my title so as to include all forms of pelvic inflammation. For I need not remind an audience such as this, that it depends on the dosage and virulence of an infection together with the resistance of the patient, whether an inflammation will pass through all its phases to suppuration. Nevertheless this trinity of problems is presented to each one of us when face to face with a pelvic inflammation, and on a correct solution depends the forecast of the progress of the case. These remarks have been suggested in some measure by the careless use of the term pyosalpinx as signifying a pelvic suppuration, and not infrequently this loose diction is used to cover all forms of tubal inflammation. But, while it may be freely admitted that tubal inflammation is the most frequent cause of pelvic suppuration there are other situations in which pus may be found in the pelvis.

Abscess of the broad ligament is not uncommon, and under certain conditions, to be discussed more fully hereafter, I believe purulent fluid will collect in the pelvis apart from a primary tubal origin. An unusual situation for pus to collect is in the uterine wall. Such an instance occurred in a lady under my care, who, when pregnant, contracted gonorrhøea. The labour was normal and her medical attendant stated that the puerperium presented nothing unusual. Within six or seven weeks after labour she began to complain of pelvic pain and had a rise of temperature, the uterus was found retrodeviated and fixed by an extensive exudate. Under rest and the usual treatment the acute symptoms subsided. The abdomen was opened with the intention of removing the infected adnexa and restoring the uterus to its normal position.

In taking a $\mathrm{V}$-shaped piece out of one uterine cornu in order thoroughly to remove the affected tube, an abscess cavity was opened

* Read before the Obstetric and Gynæcological Section, at the Australasian Medical Congress, Adelaide, S.A., 1905. 
and about 3 drachms of pus were evacuated. This cavity did not communicate with the Fallopian tube in any way, nor was there any connection with the uterine cavity. The pus from the uterine cavity was sterile, at all events no cultivations could be obtained from it. An extended experience in operative work for purulent salpingitis has led me to note how frequently the isthmic portion of the tube is free from disease when the ampulla is distended with several drachms of pus. This is the more surprising when we consider that the infection in most cases, if not in all, has travelled by direct extension from the endometrium through the tubo to its abdominal ostium. More than this, in many instances the remains of an infective endometritis can be demonstrated, so that we have the two extremities of the tract bearing evidence of disease, while the middle portion, although necessarily traversed by the infection, is apparently tree.

It may be that the anatomical relation of the isthmus of the tube to the broad ligament, with its enormous lymphatic and vascular supply, is in a better position for recovery than either the uterus or the ampullary end of the tube. When the above conditions exist a further argument is advanced for the conservative treatment of suppurative salpingitis.

It is now universally recognised that the causes of pelvic suppuration fall under three heads, and the order in which they occur as regards frequency is as follows:-

\section{Gonorrhor. \\ 2. Sepsis (parturition and abortion). \\ 3. Tuberculosis.}

Since Neoggerath years ago drew attention to the ravages wrought by the gonococcus when it once reaches the endometrium, increasing: experience has amply confirmed his views, so that it may be admitted that pelvic suppuration is chiefly due to this variety of infection. Nevertheless a not inconsiderable number of cases owe their origin to septic infection either after full time parturition or after abortion. A much smaller proportion have tuberculosis as a cause, and owing to peculiarities in pathological and clinical features form a class apart from the two preceding groups. The object of this paper is to draw attention to the part played by sepsis in bringing about a variety of pelvic inflammation, which not infrequently proceeds to the stage of suppuration.

At the outset it is important to recognise that once the genital area is infected by either the gonococcus or septic organisms, the clinical phenomena are to all intents and purposes identical. That is to say, when confronted by a case of pelvic inflammation the only 
information that will enable us to differentiate between the two groups of causes will be found in the antecedent history. At first sight this might be thought to be easily obtainable, especially when the infection manifests itself shortly after parturition; but the case just recorded will serve to point out the difficulties in arriving at a correct judgment. How much greater, therefore, must the difficulties of a correct diagnosis be increased, when the patient presents herself months, or it may be years, after confinement?

Again, it is within the experience of most of us who deal with this class of case, that pelvic suppuration by no means invariably or even frequently follows severe puerperal sepsis. In fact, it has become well recognised that it is in those instances in which the infection is of a mild degree, that subsequent pelvic mischief is to be dreaded. I have seen a woman, brought to death's door by puerperal sepsis, and yet within eighteen months delivered of a fine healthy child, and, what is more important for the purpose under discussion, making a normal recovery.

In order to make my meaning more explicit, it will perhaps be better to describe a typical case.

A married woman, the mother of several children, gives the history that all her labours have been normal, and that the convalescences, with the exception of the last, now some months ago, were also satisfactory. During the last puerperium, although not confined to bed for more than the usual time, she had for a few days some elevation of temperature and offensive lochia. She also says sho has never been quite well or strong, as she puts it, since this last confinement. Latterly the pelvic pain which hitherto only annoyed her at varying intervals has become persistent. Menstruation, when it recurred (often during lactation), was profuse, wnhealthy, and accompanied by pain. Each recurring menstrual period is now marked by a steady decline in her general health. On pelvic examination the indisputable signs of tubal inflammation can be discovered, but without any extensive exudate. Not infrequently the uterus will be retroposed and may deceive the unwary by its apparent mobility. Thus far the case is common enough, but a further stage in not a few instances is reached. Under favourable circumstances the mild, slowly developing infection will wear itself out and the inflammatory process come to an end, leaving the tube occluded at the abdominal end and perhaps adherent, or at all events fixed in close proximity to intestine. Under such circumstances it is conceivable that it only requires something to lower the resistance of the tissues, e.g., influenza, exhausting fevers, etc., in order that the colon 
bacillus may pass from the intestine along the line of adhesion and re-infert the damaged tube. That this does take place when the appendix vermiformis becomes attached to the Fallopian tube under such conditions is almost unanimously admitted. Mr. Bland-Sutton recently reported a case where the appendix vermiformis opened into an occluded Fallopian tube and discharged pus into the ampulla. The essential point, in the majority of instances, is the injury done to the tube by a prior infection from the uterus.

In looking over the histories of a number of cases of chronic salpingitis it was noticeable how many dated their ill-health from an antecedent abortion. Nor was this altogether confined to those patients who, either from lack of opportunity or careless ignorance, failed to obtain medical assistance. And in not a few the information was rouchsafed that "the Doctor "curated." In these it might be permissible to doubt if the cure was not, at all events, as bad as the disease, seeing that the tubes became affected all the same.

When it is recollected that the pathological events which accompany abortion-the hæmorrhage, tearing of foetal envelopes, etc.,-all invite sepsis, it is not to be wondered at that a mild infection ensues with its usual sequelæ. And it is quite conceivable that a failure to maintain an aseptic technique in the treatment of an incomplete abortion will result in an infection of the upper generative tract, notwithstanding an apparently good recovery at the time. It may be asserted as a fairly general rule that the morbidity resulting from an abortion works its greatest mischief at a late date. Instances such as are here mentioned must be familiar to everyone in family practice who cares to follow up obstetric histories by a careful gynæcological inquiry. Of course it is not intended to imply that every abortion is necessarily followed by a salpingitis, but merely to point out how favourable the phenomena of abortion are to the inception of a mild sepsis. One might as well say that in the preantiseptic midwifery days every parturient was doomed to sepsis; those of us who experienced those times will remember the frequent outbursts of "puerperal fever" and also its mortality.

While directing your attention to some of the less obvious causes of pelvic inflammation, it may be of some profit to see what lessons can be drawn from them. Let us take first the cases following on a mild sepsis occurring during the puerperium. If it once be recognised that such serious lesions as salpingitis and more or less pelvic peritonitis do sometimes owe their origin to a mild degree of infection arising during the recovery from parturition, it is obvious that more importance will be attached to abnormalities of the puerperium even though they be slight and temporary. If practitioners would under- 
take to mould maternal opinion in the direction of submitting to systematic pelvic examination instituted after the period of normal involution had passed, a step forward would be made in fighting so insidious a foe. Even if only those cases in which slight irregularities occurred during the puerperium were subsequently examined, much future trouble would be averted. A careful aseptic curettage, with the establishment of good drainage from the uterine cavity, at this stage, would undoubtedly be efficacious in preventing many a subsequent dangerous and mutilating operation.

The second lesson if short, is emphatic. It applies to the emptying of the uterus in abortion at the very earliest opportunity. If the principles which govern modern gynæcological practice are correct no sound argument can be adduced in favour of waiting on nature to complete the abortion. No doubt nature is quite capable of so simple a matter as evacuating the uterus, but as our knowledge of modern pathology tells us, she calls to her aid certain agents whose actions she cannot always control.

The third lesson to be drawn from the consideration of these few points is that an apparently quiescent salpingitis may become reinfected through adhesions to intestine. The woman with such a pelvic condition is never safe while she retains her damaged tubes.

Reflection on these cases of pelvic inflammation brings the conviction that many are in a sense preventable, and moreover that we possess two lines of defence. The first entails greater care during parturition as to aseptic technique and closer observation of the puerperium whereby the incidence of a mild sepsis may be prevented. The second line may be regarded as establishing the eustom of postpuerperal examination, especially where any irregularity in temperature, pulse rate, and condition of the lochia has been noted. By these means I believe we shall help to bring gynæcology into line with other departments of medical practice where the prevention of gross and other irreparable disease is the ideal aim.

Brilliant as the achievements of gynæcological surgery have been during the past twenty years in saving life and restoring the invalid to health, it must not be forgotten that these results have been attained by methods crude in themselves and destructive of physiological function. That this is recognised in some measure by a number of pelvic surgeons, the various plastic procedures on damaged tubes and resections of ovaries bear witness. These so-called conservative operations must at best be classified as experimental, and we have to confess that at present we lack any systematized scheme calculated to restore the physiological integrity of these important organs. Is not the time approaching when it will be incumbent on us to seek out the prime causes of the gross lesion and thereby prevent destruction of physiological function? 